\title{
NUCLEAR-STRUCTURE DEPENDENCE OF THE FUSION CROSS SECTION FOR HEAVY IONS
}

\author{
M. LOZANO and G. MADURGA \\ Departamento de Fiśsica Atómica y Nuclear, Universidad de Sevilla, Spain
}

Received 7 November 1979

\begin{abstract}
The energy dependence of the fusion cross section and its maximum value are well predicted, for a wide range of nuclei and energies, by introducing information on the nuclear matter density distribution into a simple formula.
\end{abstract}

In the last few years many measurements of the fusion cross section for heavy ions have been reported. Refs. [1-6] are some examples. At low energies the behavior of $\sigma_{\mathrm{fus}}(E)$ is thought to be dominated by the interaction barrier $V\left(R_{\mathrm{B}}\right)$. A simple parametrization, based on semiclassical or classical ideas, yields the relation [1]:

$\sigma_{\mathrm{fus}}=\pi R_{\mathrm{B}}^{2}\left[1-V\left(R_{\mathrm{B}}\right) / E\right]$,

$E$ being the center-of-mass energy. This expression is considered by Glas and Mosel [7] a limiting case of a more elaborated theory in which the critical distance or "point of no return" becomes smaller at higher energies. Expression of $\sigma_{\mathrm{fus}}(E)$, as a function of the mass $A$ and charge $Z$ of the colliding nuclei, have been obtained from the proximity potential [8] or from empirical potentials fitted to the data [9].

Recent experiments, nevertheless, point to a $\sigma_{\text {fus }}(E)$ dependence on the nuclear structure of the particular colliding nuclei $[3,6]$ : an oscillatory behavior of $\sigma_{\mathrm{fus}}(E)$ around the maximum is found in some cases $\left({ }^{12} \mathrm{C}+{ }^{12} \mathrm{C},{ }^{12} \mathrm{C}+16 \mathrm{O}, 16 \mathrm{O}+16 \mathrm{O}\right)$. Also a clear enhancement of the maximum value of the fusion cross section, $\sigma_{\mathrm{fus}}^{\max }$, is noticed when one or two nucleons are first added to a new major shell.

Horn and Ferguson [10] have introduced into their very simple empirical formula some information on the target and projectile structure by means of a "contact distance", $b$, which is taken to be the sum of the radii at $1.35 \%$ of the central charge density. Eq. (1) is changed into: $\sigma_{\text {fus }}(E)=\pi \rho(\rho-D)$,

where $D=Z_{1} Z_{2} e^{2} / E$ is the minimum distance in a head-on collision between point-like particles, only the Coulomb potential has been explicitly considered, and a varying parameter $\rho$ has been introduced instead of $R_{\mathrm{B}}$ in order to simulate the effects of the nuclear interaction. Over a range of energies from 1.2 times the Coulomb barrier up to beyond the energy $E_{1}$ for which Bass [9] predicts the onset of tangential friction, good agreement with empirical data is found if the parameter $\rho$ differs from the above-mentioned contact distance by a term proportional to $E$ :

$\rho=b+m E$.

The coefficient $m=\mathrm{d} \rho / \mathrm{d} E$ depends on the compound nucleus mass and is given [10] by the equation $m^{-1}$ $=18\left[2.23-\left(A_{1}+A_{2}\right)^{1 / 3}\right] \mathrm{MeV} / \mathrm{fm}$.

In this letter we modify Horn and Ferguson's prescription and relate the contact distance $b$ to the nuclear mass density rather than to the charge density. We do so because the former should be of greater significance, and particularly because in some similar problems we have succeeded in reproducing small departures from a mere $A^{1 / 3}$-behavior by using information on the nuclear matter density at far out distances. So the critical radii for alpha-particle elastic scattering, as analyzed by Badawy et al. [11], have been related to the distance where the density is 0.002 nucleon $\mathrm{fm}^{-3}[12]$. In the latter work we used the nuclear density distribution calculated as the sum of singleparticle wavefunctions weighted by appropriate occu- 
pation numbers. A detailed account of the method is given in ref. [13] and summarized in ref. [12]. Ideally "experimental" information such as charge rms radii, fractional occupation numbers and single-particle centroid energies, should be fed into the model for a suitable choice of the parameters so as to optimize the description of each nuclide. Nevertheless all these data are not always available and we have shown [12] that the overall systematic fit of the model parameters, found by Bear and Hodgson [14], gives values of the density tail essentially equivalent to those of other sets of parameters chosen "ad hoc" for each individual nucleus.

Consequently the prescription of ref. [14] has been used to obtain systematic nuclear matter density distributions. With the help of them we also have been able [15] to improve considerably the general optical potential for heavy-ion elastic scattering proposed by Christensen and Winther [16] .

These two encouraging experiences support the hope that the above description of the nuclei can be used for a more reasonable determination of the contact distance $b$ of eq. (3). By introducing into eq. (2) the measured values of $\sigma_{\text {fus }}$ at different energies, the "experimental" values of $\rho$ are seen to be linear in $E$, and the $E \rightarrow 0$ limits for different pairs of nuclei suggest as a good choice for the contact distance:

$b=$ sum of radii at density 0.002 nucleon $/ \mathrm{fm}^{3}$.

Table 1 gives the values of such radii for the nuclides

Table 1

Distance $R$ from the center of the nucleus where the nuclear matter density is 0.002 nucleon $/ \mathrm{fm}^{3}$, computed as in refs. $[13,14]$. The sum of two such radii is the contact distance $b$ to be used in eq. (3) for the computation of the corresponding ofus

\begin{tabular}{llll}
\hline Nucleus & $R(\mathrm{fm})$ & Nucleus & $R(\mathrm{fm})$ \\
\hline${ }^{12} \mathrm{C}$ & 4.51 & ${ }^{27} \mathrm{Al}$ & 5.30 \\
${ }^{13} \mathrm{C}$ & 4.58 & ${ }^{32} \mathrm{~S}$ & 5.58 \\
${ }^{14} \mathrm{~N}$ & 4.62 & ${ }^{35} \mathrm{Cl}$ & 5.66 \\
${ }^{15} \mathrm{~N}$ & 4.66 & ${ }^{40} \mathrm{Ca}$ & 5.79 \\
${ }^{16} \mathrm{O}$ & 4.70 & ${ }^{48} \mathrm{Ti}$ & 6.04 \\
${ }^{17} \mathrm{O}$ & 4.79 & ${ }^{54} \mathrm{Fe}$ & 6.20 \\
${ }^{18} \mathrm{O}$ & 4.88 & ${ }^{56} \mathrm{Fe}$ & 6.31 \\
${ }^{19} \mathrm{~F}$ & 4.93 & ${ }^{62} \mathrm{Ni}$ & 6.53 \\
${ }^{24} \mathrm{Mg}$ & 5.19 & $116 \mathrm{Sn}$ & 7.60 \\
${ }^{26} \mathrm{Mg}$ & 5.26 & $124 \mathrm{Sn}$ & 7.76 \\
\hline
\end{tabular}

used in this work and computed by the method just described. Now the correct slope $m$ of $\rho(E)$, as a function of the compound nucleus mass $A=A_{1}+A_{2}$, is such that

$m^{-1}=21\left(2.44-A^{1 / 3}\right) \mathrm{MeV} / \mathrm{fm}$.

Fig. 1 and 2 show experimental data on the fusion cross section for 15 pairs of nuclei at several c.m. energies and the prediction of eqs. (2) to (5). The agreement is very good.

This model cannot reproduce the oscillatory behavior of $\sigma_{\text {fus }}(E)$ with resonance-like bumps for some special cases, which is not well understood as yet and could be attributed to other competing channels. It does however show a large enhancement of $\sigma_{\text {fus }}^{\max }$ for ${ }^{12} \mathrm{C}$ with ${ }^{17} \mathrm{O}$ and ${ }^{18} \mathrm{O}$ as compared to ${ }^{16} \mathrm{O}$, which appears in experimental data, and a moderate rise for ${ }^{15} \mathrm{~N}$ relative to ${ }^{14} \mathrm{~N}$. Fig. 3 shows the measured maximum value of the fusion cross section for ${ }^{12} \mathrm{C}$ and ${ }^{16} \mathrm{O}$ with several light or medium-weight nuclei compared with our predictions as well as those obtained with Horn and Ferguson's prescription and with Bass' model.

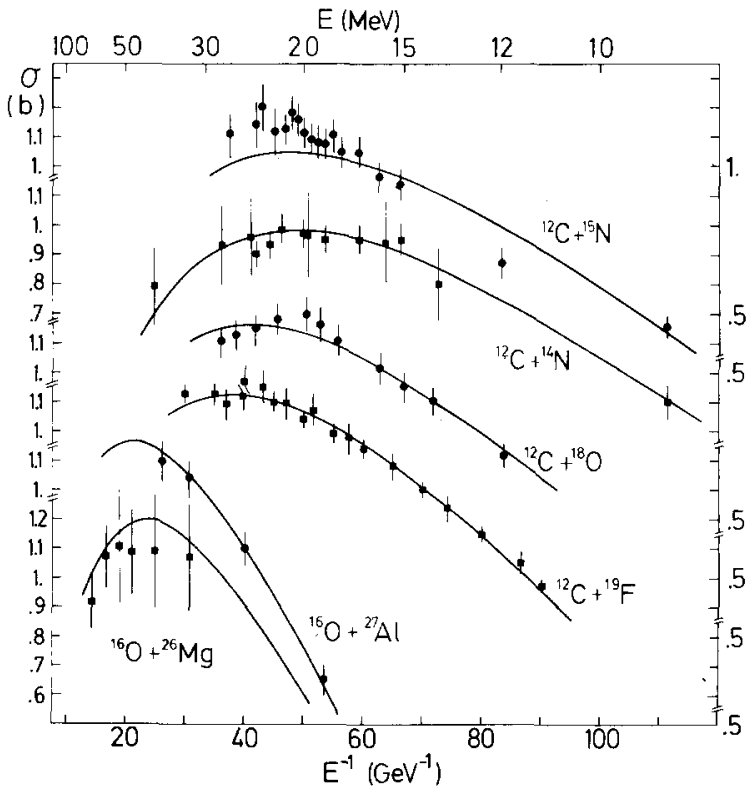

Fig. 1. Fusion cross section as a function of $E_{\mathrm{cm}}^{-1}$ for several projectiles on ${ }^{12} \mathrm{C}$ and for ${ }^{16} \mathrm{O}$ on ${ }^{27} \mathrm{Al}$ and ${ }^{26} \mathrm{Mg}$. Experimental values from refs. [4-6] (black circles or squares, for alternate pairs) are compared to the predictions of this work (solid lines). 


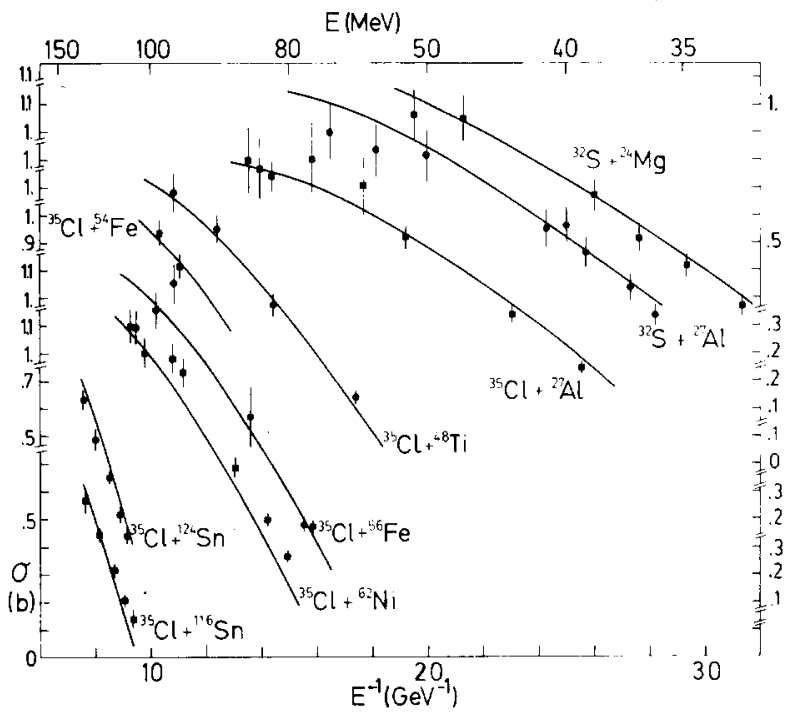

Fig. 2. Same as fig. 1 for heavier pairs. Data with ${ }^{32} \mathrm{~S}$ as the projectile are from ref. [3], those with ${ }^{35} \mathrm{Cl}$ as the projectile are from refs. $[1,2]$.

We believe that our contribution improves significantly Horn and Ferguson's model and gives a good prescription to predict fusion cross sections for a wide range of nuclei and energies. This success once more confirms the validity of our nuclear density distributions in the surface region.

We are grateful to D.G. Kovar and J.P. Schiffer and to D. Horn and A.J. Ferguson for sending us data prior to publication, to P.E. Hodgson for many constructive discussions, and to the Instituto de Estudios Nucleares, Madrid, for financial help.

\section{References}

[1] H.H. Gutbrod, W.G. Winn and M. Blann, Nucl. Phys. A213 (1973) 267.

[2] W. Scobel, H.H. Gutbrod, M. Blann and A. Mignerey, Phys. Rev. C14 (1976) 1808.

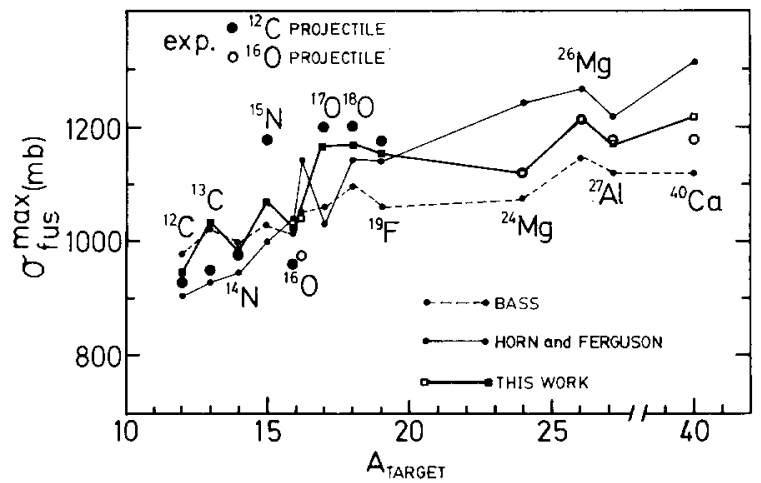

Fig. 3. Experimental maximum fusion cross section for ${ }^{12} \mathrm{C}$ (black large circles) or ${ }^{16} \mathrm{O}$ (open large circles) with several nuclei. The predictions of this work (thick line with squares) are compared to those of ref. [10] (thin line and dots) and ref. [9] (dashed line and dots).

[3] P. Sperr et al., Phys. Rev. Lett. 37 (1976) 321.

[4] R.G. Stokstad et al., Phys. Lett. 70B (1977) 289.

[5] D. Horn, A.J. Ferguson and O. Haüser, Nucl. Phys. A311 (1978) 238; and to be published.

[6] D.G. Kovar et al., Phys. Rev. C20 (1979), to be published; D.G. Kovar et al., in: Proc. Intern. Conf. on Nuclear structure (Tokyo, Japan, 1977), ed. Organizing Committee (International Academic Printing Co., Tokyo, 1977) p. 660.

[7] D. Glas and U. Mosel, Nucl. Phys. A237 (1975) 429.

[8] J.R. Birkelund, J.R. Huizenga, J.N. Dee and D. Sperber, Phys. Rev. Lett. 40 (1978) 1123; L.C. Vaz and J.M. Alexander, Phys. Rev. C18 (1978) 2152.

[9] R. Bass, Phys. Rev. Lett. 39 (1977) 265.

[10] D. Horn and A.J. Ferguson, Phys. Rev. Lett. 41 (1978) 1529 .

[11] I. Badawy et al., Phys. Rev. C17 (1978) 978.

[12] M. Lozano, G. Madurga and P.E. Hodgson, Phys. Lett. 82B (1979) 170.

[13] F. Malaguti, E. Verodini, A. Uguzzoni and P.E. Hodgson, Nucl. Phys. A297 (1978) 287.

[14] R. Bear and P.E. Hodgson, J. Phys. G4 (1978) L287.

[15] M. Lozano, G. Madurga and P.E. Hodgson, University of Seville report FANSE-79-08.

[16] P.R. Christensen and Aa. Winther, Phys. Lett. 65B (1976) 19. 